\title{
A Technique for Extracting Physiological Parameters and the Required Input Function Simultaneously from PET Image Measurements: Theory and Simulation Study
}

\author{
Dagan Feng, Senior Member, IEEE, Koon-Pong Wong, Chi-Ming Wu, and Wan-Chi Siu, Senior Member, IEEE
}

\begin{abstract}
Positron emission tomography (PET) is an important tool for enabling quantification of human brain function. However, quantitative studies using tracer kinetic modeling require the measurement of the tracer time-activity curve in plasma (PTAC) as the model input function. It is widely believed that the insertion of arterial lines and the subsequent collection and processing of the biomedical signal sampled from the arterial blood are not compatible with the practice of clinical PET, as it is invasive and exposes personnel to the risks associated with the handling of patient blood and radiation dose. Therefore, it is of interest to develop practical noninvasive measurement techniques for tracer kinetic modeling with PET. In this paper, a technique is proposed to extract the input function together with the physiological parameters from the brain dynamic images alone. The identifiability of this method is tested rigorously by using Monte Carlo simulation. The results show that the proposed method is able to quantify all the required parameters by using the information obtained from two or more regions of interest (ROI's) with very different dynamics in the PET dynamic images. There is no significant improvement in parameter estimation for the local cerebral metabolic rate of glucose (LCMRGlc) if the number of ROI's are more than three. The proposed method can provide very reliable estimation of LCMRGlc, which is our primary interest in this study.
\end{abstract}

Index Terms - Modeling, noninvasive measurement, positron emission tomography (PET), simulation.

\section{INTRODUCTION}

$\mathbf{T}$ HE USE of the tracer kinetic modeling technique in positron emission tomography (PET) enables quantification of physiological and biochemical processes in humans in vivo. Measurements of the tracer time-activity curves in both plasma (PTAC) and tissue (TTAC) are required to estimate the physiological parameters, i.e., to fit the parameters of certain compartmental models by using PTAC and TTAC as the model

Manuscript received April 25, 1997; revised December 3, 1997. This work was supported by RGC, NHMRC, and ARC grants.

D. Feng and K.-P. Wong are with the Biomedical and Multimedia Information Technology Group, Department of Computer Science, The University of Sydney, Sydney, 2006 Australia, and the Department of Electronic Engineering, The Hong Kong Polytechnic University, Hung Hom, Hong Kong (e-mail: feng@cs.usyd.edu.au).

C.-M. Wu and W.-C. Siu are with the Department of Electronic Engineering, The Hong Kong Polytechnic University, Hung Hom, Hong Kong.

Publisher Item Identifier S 1089-7771(97)09406-5. input and output functions, respectively. The input PTAC are usually obtained by taking blood samples invasively from an artery (or arterialized vein) [1], [2].

It is generally accepted that the insertion of arterial lines and the subsequent collection and processing of arterial blood is not compatible with the practice of clinical PET, as it requires extra personnel and processing time, exposes the patient to the risks associated with the insertion of an arterial line, and exposes personnel to the risks associated with the handling of patient blood and increased radiation from proximity to the patient [3]. Therefore, it is of interest to develop practical noninvasive techniques for tracer kinetic modeling with PET.

Watabe et al. recently presented a method for the pixel-bypixel quantification of regional cerebral blood flow (rCBF) by using Oxygen-15 water [4]. They defined two regions as grey matter and whole brain, respectively. Two equations representing two regions derived from the CBF model were utilized for eliminating blood terms. The method can accurately detect relative changes in CBF, which is mainly restricted to brain activation studies. Carson et al. presented a method for absolute CBF determination without a measured input function by also using Oxygen-15 water and PET [5]. They treated the unmeasured $M$-discrete blood samples as the $M$-unknown parameters to be estimated during the modeling process together with the $N$-pixel blood flow parameters. In other words, $N+M$ parameters would be estimated from the $M$-scan frames, with the total number of measurements being $N \times M$. If the number of scan frames is large, the computational complexity is very high. Moreover, this method is difficult to extend to the tracer fluoro-deoxy-glucose (FDG) for glucose metabolism or other general second-order systems, as many discrete PTAC sample values are involved.

In cardiac studies, input functions can be derived noninvasively from regions of interest (ROI's) drawn on the vascular structures, such as the left ventricle (LV), visualized on the PET images [6]. However, the input functions obtained in this manner are extremely noisy and severely contaminated by the radioactivity from adjacent tissue. The direct use of such input functions in modeling increases the statistical uncertainties in the estimated model parameters [7]. Furthermore, the vascular structures visualized during FDG brain imaging are insufficient for this method. 


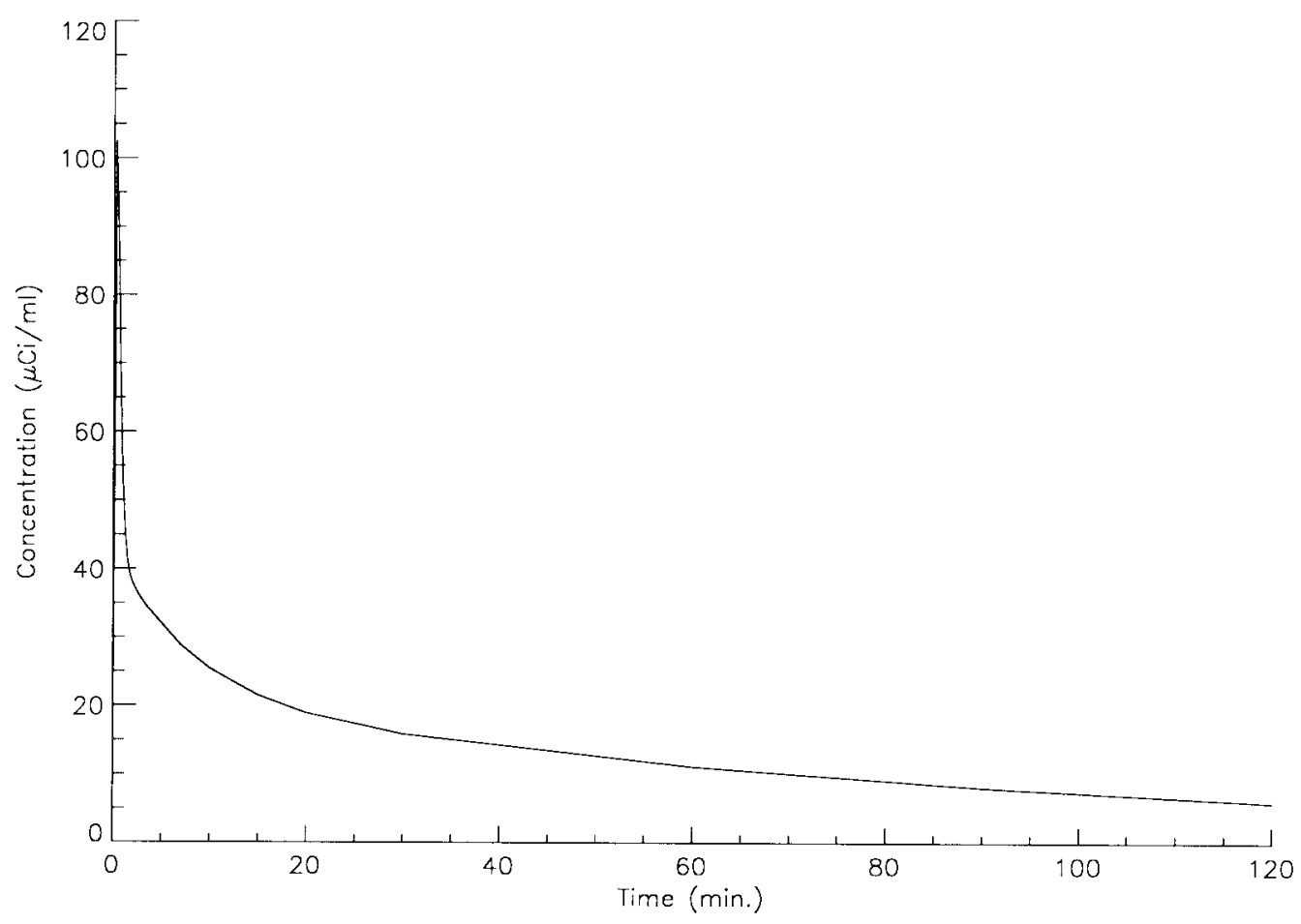

Fig. 1 Plot of input function used in the simulation study. Data were obtained from actual human study.

With the aim of solving the noise and spillover problems in noninvasive measurements, various PTAC models have been studied based on the FDG experimental data obtained from blood samples. The use of a model for PTAC to filter the noisy input function during the physiological parameter estimation procedure can significantly improve estimation accuracy [8], [9]. Among these studies of PTAC models, a fourth-order model with a pair of repeated eigenvalues can best describe the complicated behavior of PTAC in PET studies [9].

There have been a number of methods proposed for the simplification of FDG studies. One was proposed by Hutchins et al. for an efficient quantification of FDG studies by using single-scan frame for the estimation of local cerebral metabolic rate of glucose (LCMRGlc) [10], while some studies on the noninvasive measurement of input function have also been reported in the literature [11], [12]. In this paper, a new technique is proposed to extract the input function together with the physiological parameters from brain dynamic images either with or without late venous blood samples. The identifiability of this method is tested by using Monte Carlo simulation. We used the FDG three-compartment model as an example to investigate the technique.

\section{THEORY}

\section{A. Plasma Time-Activity Input Function}

The PTAC $c_{p}^{*}(t)$ is used as the input function in modeling the tracer kinetic model. PTAC is usually represented by a sequence of blood samples. A model for PTAC was proposed in [9], which has been used in optimizing the input-function sampling schedule [13], in correcting the contaminated input function [14], [15], and in using the indirectly measured input functions [16]. We are going to use this model here to extract the input function from the overall PET measurements. The mathematical expression for this PTAC model without the delay factor is given by

$$
c_{p}^{*}(t)=\left(A_{1} t-A_{2}-A_{3}\right) e^{\lambda_{1} t}+A_{2} e^{\lambda_{2} t}+A_{3} e^{\lambda_{3} t}
$$

where $\lambda_{1}, \lambda_{2}$, and $\lambda_{3}$ (in $\min ^{-1}$ ) are the eigenvalues of the model and $A_{1}$ (in $\mu \mathrm{Ci} / \mathrm{ml} / \mathrm{min}$ ), $A_{2}$ and $A_{3}$ (in $\mu \mathrm{Ci} / \mathrm{ml}$ ) are the coefficients of the model. A typical PTAC curve is shown in Fig. 1.

\section{B. FDG Model}

The three-compartment FDG model (shown in Fig. 2) was originally proposed by Sokoloff et al. [17] and further extended by Huang et al. [1] and Phelps et al. [2]. This threecompartment model is going to be used in our study as an example to illustrate the new method. The differential equations describing the kinetics of FDG for the three-compartment model are given by

$$
\begin{aligned}
\frac{d}{d t} c_{e}^{*}(t) & =k_{1}^{*} c_{p}^{*}(t)-\left(k_{2}^{*}+k_{3}^{*}\right) c_{e}^{*}(t)+k_{4}^{*} c_{m}^{*}(t) \\
\frac{d}{d t} c_{m}^{*}(t) & =k_{3}^{*} c_{e}^{*}(t)-k_{4}^{*} c_{m}^{*}(t) \\
c_{i}^{*}(t) & =c_{e}^{*}(t)+c_{m}^{*}(t)
\end{aligned}
$$

where $k_{1}^{*}-k_{4}^{*}$ are the rate constants, $c_{p}^{*}(t)$ is the FDG concentration in plasma, $c_{e}^{*}(t)$ is the free FDG in tissue, $c_{m}^{*}(t)$ is the concentration of FDG-6-phosphate in tissue, and $c_{i}^{*}(t)$ is the 


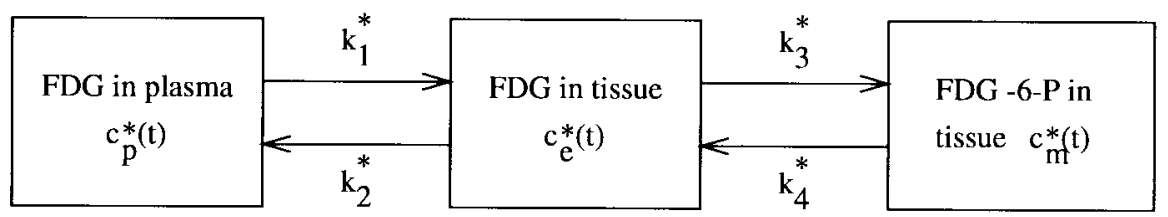

Fig. 2. Three-compartment FDG model used in the simulation study.

total tissue activity. The solution of $c_{i}^{*}(t)$ is given by

$$
\begin{gathered}
c_{i}^{*}(t)=\left\{\frac{k_{1}^{*}}{\alpha_{2}-\alpha_{1}}\left[\left(k_{4}^{*}-\alpha_{1}\right) e^{-\alpha_{1} t}+\left(\alpha_{2}-k_{4}^{*}\right) e^{-\alpha_{2} t}\right]\right. \\
\left.+\frac{k_{1}^{*} k_{3}^{*}}{\alpha_{2}-\alpha_{1}}\left(e^{-\alpha_{1} t}-e^{-\alpha_{2} t}\right)\right\} \otimes c_{p}^{*}(t)
\end{gathered}
$$

where

$$
\alpha_{1,2}=\frac{k_{2}^{*}+k_{3}^{*}+k_{4}^{*} \mp \sqrt{\left(k_{2}^{*}+k_{3}^{*}+k_{4}^{*}\right)^{2}-4 k_{2}^{*} k_{4}^{*}}}{2}
$$

and $\otimes$ denotes the operation of convolution. Equation (5) can then be described by the macroparameters of the model as

$$
c_{i}^{*}(t)=\left(B_{1} e^{-L_{1} t}+B_{2} e^{-L_{2} t}\right) \otimes c_{p}^{*}(t)
$$

where

$$
\begin{array}{ll}
B_{1}=\frac{k_{1}^{*}}{\alpha_{2}-\alpha_{1}}\left(k_{3}^{*}+k_{4}^{*}-\alpha_{1}\right), & L_{1}=\alpha_{1} \\
B_{2}=\frac{k_{1}^{*}}{\alpha_{2}-\alpha_{1}}\left(\alpha_{2}-k_{3}^{*}-k_{4}^{*}\right), & L_{2}=\alpha_{2}
\end{array}
$$

are the macroparameters in the FDG model and $c_{p}^{*}(t)$ is the PTAC.

In dynamic PET studies, the calculation of the rate constants requires the TTAC generated from sequential tomographic images. The total activity of tracer in tissue, however, includes the activity in the vascular space of tissue, which is very high in the early time of the scanning period. The contribution of the activity in the vascular space of tissue can cause significant errors in the estimation of the model rate constants and the derived value of the local cerebral metabolic rate of glucose (LCMRGlc) [18]. In view of this, a cerebral blood volume (CBV) term is included in the FDG model to account for this effect and to model the in vivo situation. The observed total tissue activity, $c_{T}^{*}(t)$, is

$$
c_{T}^{*}(t)=(1-\mathrm{CBV}) \cdot c_{i}^{*}(t)+\mathrm{CBV} \cdot c_{p}^{*}(t)
$$

where $B_{1}, L_{1}, B_{2}$, and $L_{2}$ are defined above. With the measurements of both PTAC $\left[c_{p}^{*}(t)\right]$ and TTAC $\left[c_{T}^{*}(t)\right],(8)$ can be used to estimate $B_{1}, L_{1}, B_{2}, L_{2}$, and $\mathrm{CBV}$ via the nonlinear regression method. Once the macroparameters are estimated, the corresponding microparameters $\left(k_{1}^{*}-k_{4}^{*}\right)$ can thus be obtained by the following simple algebraic relations:

$$
\begin{aligned}
k_{1}^{*} & =B_{1}+B_{2} \\
k_{2}^{*} & =\frac{B_{1} L_{1}+B_{2} L_{2}}{B_{1}+B_{2}} \\
k_{3}^{*} & =\frac{B_{1} L_{2}+B_{2} L_{1}}{B_{1}+B_{2}}-\frac{L_{1} L_{2}\left(B_{1}+B_{2}\right)}{B_{1} L_{1}+B_{2} L_{2}} \\
k_{4}^{*} & =\frac{L_{1} L_{2}\left(B_{1}+B_{2}\right)}{B_{1} L_{1}+B_{2} L_{2}}
\end{aligned}
$$

and their formulation are the same as those given by [1] and [2]. LCMRGlc can then be calculated by the following formula $[2]$ :

$$
\text { LCMRGIc }=\frac{1}{\operatorname{LC}} \frac{k_{1}^{*} k_{3}^{*}}{k_{2}^{*}+k_{3}^{*}} c_{p}
$$

where LC is the lumped constant that embodies the difference between FDG and glucose in transportation and phosphorylation, and it is usually assumed to be constant. $c_{p}$ denotes the "cold" glucose concentration in plasma, which can be obtained from external blood sampling. Further details and assumptions about LC can be found in [1]. Estimation of $k_{1}^{*}-k_{4}^{*}$, the $\mathrm{CBV}$, the combined parameter $K=k_{1}^{*} k_{3}^{*} /\left(k_{2}^{*}+k_{3}^{*}\right)$, and the determination of the PTAC are our primary interest in this study.

\section{PET Measurements}

The PET measurements are the accumulated counts of photons for each particular time interval $I_{k}(k=1,2, \cdots, M)$. Therefore, the PET measurements obtained during a sequence of time interval $I_{k}$ are affected by the length of the corresponding time intervals. In this paper, the average value over the length of the scanning interval is used as $c_{T}^{*}\left(t_{k}^{\prime}\right)$, which equals

$$
c_{T}^{*}\left(t_{k}^{\prime}\right)=\frac{1}{\Delta t_{k}} \int_{t_{k-1}}^{t_{k}} c_{T}^{*}(t) d t
$$

where $t_{k}^{\prime}=\left(t_{k-1}+t_{k}\right) / 2$ and $\Delta t_{k}=t_{k}-t_{k-1}$ is the length of scanning interval $I_{k}$.

\section{PET Measurement Variance Structure}

It is very important to have an appropriate variance structure to account for the effects of the length of scanning time on the measurement. As the measurements in PET are actually the average of counts acquired during the scanning intervals, the variances of the measurement noise are proportional to the radioactivity concentrations and inversely proportional to the lengths of the scanning intervals [8], [14]. Therefore, the variance structure of PET measurement can be described as

$$
\sigma^{2}\left(t_{k}^{\prime}\right)=\frac{\alpha \times c_{T}^{*}\left(t_{k}^{\prime}\right)}{\Delta t_{k}}
$$

where $\sigma^{2}\left(t_{k}^{\prime}\right)$ are the corresponding variances of the output function measurements at midtime $t_{k}^{\prime}$ of the $k$ th scanning interval and $\alpha$ is the proportionality constant that determines the noise level in the measurement. 


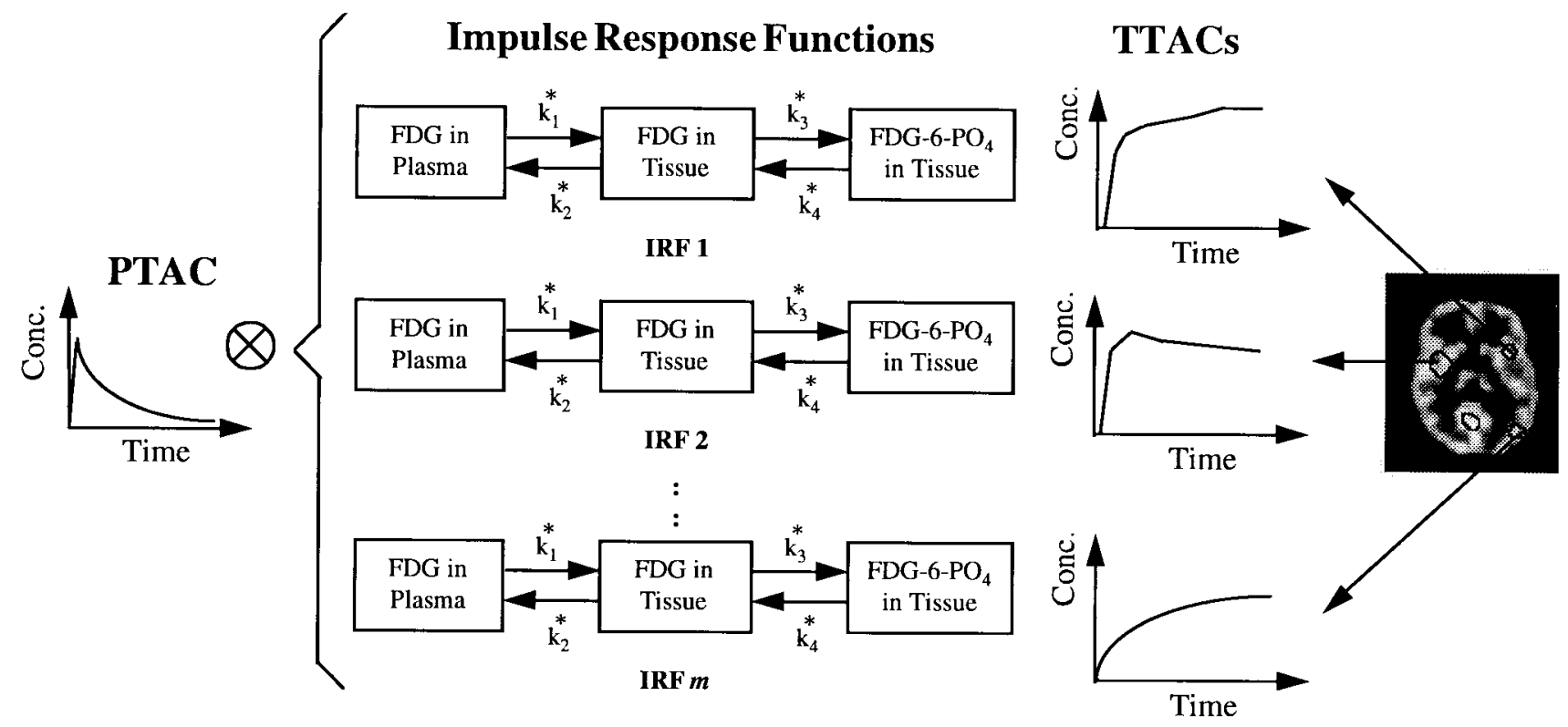

Fig. 3. TTAC's from the images are used to fit the convolution of the PTAC with the tissue impulse-response functions, where $\otimes$ is the convolution operator. From the fitted-rate constant parameters in the FDG model, the physiological parameters corresponding to the local regions can be obtained.

\section{E. The Proposed New Method}

For a given output curve of a linear time-invariant system, if the system transfer function is known, we can use deconvolution techniques to obtain the input function, or if the input function is known, we can estimate the transfer function. Nevertheless, we cannot obtain the input function and the system transfer function simultaneously from the single output function. In PET dynamic studies, multiple output functions can be obtained from different ROI's, as shown in Fig. 3. These output functions or measurements are the convolution of the physiological impulse-response functions (IRF's) corresponding to the local regions with the same input function (PTAC). The PTAC and physiological parameters may thus be estimated simultaneously from $t w o$ or more output curves (TTAC's) sampled from various regions in the dynamic images.

Although using more ROI information may provide more reliable parameter estimation, the number of parameters to be estimated and the computational complexity will also be increased. Therefore, we need to find how many ROI's are adequate to be used in parameter estimation. In the following study, we compare the identifiability and reliability for parameter estimation using the information obtained from two, three, and four ROI's. In addition, we are going to show the results when only TTAC's are used for parameter estimation and what happens when a couple of blood samples are used to help quantification of parameters in IRF's and PTAC.

\section{SimUlation STUDY}

\section{A. Generation of Simulation Data}

Computer simulation was performed to test the identifiability and reliability of the proposed method. The PTAC $c_{p}^{*}(t)$ was generated from (1) with the average values obtained in [9]: $A_{1}=851.1225, A_{2}=21.8798, A_{3}=20.8113, \lambda_{1}=$ $-4.1339, \lambda_{2}=-0.1191$, and $\lambda_{3}=-0.0104$, sampled at $0.25,0.5,0.75,1,1.25,1.5,1.75,2,2.5,3,3.5,7,10,15$, 20, 30, 60, 90, and 120 min. Fig. 1 shows the input function generated by using the above set of parameters and sampling schedule. The TTAC's $c_{T}^{*}(t)$ were calculated according to (8) by using $c_{p}^{*}(t)$, given above, and four sets of typical microparameters of the FDG models obtained in human study [18], as shown in Table I. For each TTAC, there were 22 scanning intervals, consisting of $10 \times 12$-s scans, $2 \times 0.5$ min scans, $2 \times 1$-min scans, $1 \times 1.5$-min scan, $1 \times 3.5$-min scan, $2 \times 5$-min scans, $1 \times 10$-min scan, and $3 \times 30$-min scans. A pseudorandom number generator was used to generate the Gaussian noise added on to $c_{T}^{*}(t)$, according to the error variance formula (11), in which $\alpha$ was set to $0.1,0.5,1.0,2.0$, and 4.0, respectively.

\section{B. Implementation of the New Method}

Use of Blood Samples: In the PTAC model, the last two parameters $A_{3}$ and $\lambda_{3}$ will dominate the tail of the PTAC function (i.e., when $t$ is greater than a large constant or near the end of the scanning period). In practical applications, these two parameters can be determined by fitting two or more blood samples measured near the end or after the image scanning, as blood samples are needed to measure the cold glucose concentration anyway. Thus, the computational complexity for simultaneous fitting of the parameters of the PTAC and the IRF's can be reduced, while the numerical identifiability of the parameters can also be improved. In our simulation, these two parameters were kept constants to emulate the act of blood sampling of the two blood samples at time instants for $t=90$ and $t=120 \mathrm{~min}$, respectively, i.e., the last two time instants of the PTAC sampling schedule given above. On the other 
TABLE I

Set of Rate Constants Used in the Simulation Study

\begin{tabular}{c|cccc|c}
\hline & \multicolumn{5}{|c}{ Rate constants } \\
Structure & $\begin{array}{c}k_{1}^{*} \\
(\mathrm{ml} / \mathrm{min} / \mathrm{g})\end{array}$ & $\begin{array}{c}k_{2}^{*} \\
\left(\mathrm{~min}^{-1}\right)\end{array}$ & $\begin{array}{c}k_{3}^{*} \\
\left(\mathrm{~min}^{-1}\right)\end{array}$ & $\begin{array}{c}k_{4}^{*} \\
\left(\mathrm{~min}^{-1}\right)\end{array}$ & $\begin{array}{c}C B V \\
(\mathrm{ml} / 100 \mathrm{~g})\end{array}$ \\
\hline Grey matter & 0.041 & 0.119 & 0.064 & 0.0075 & 5.80 \\
White matter & 0.023 & 0.073 & 0.034 & 0.0098 & 2.50 \\
Tumor & 0.028 & 0.087 & 0.047 & 0.0084 & 4.40 \\
Hemisphere & 0.034 & 0.112 & 0.061 & 0.0098 & 5.00 \\
\hline
\end{tabular}

hand, the effects of ignoring these two blood samples to the quantification of the parameters in IRF's and PTAC have also been studied by simultaneous fitting of the parameters in PTAC and IRF's without the two blood samples, and the results are also compared to those obtained with the blood samples to see the changes in precision and accuracy of the estimated parameters in IRF's and PTAC.

Cost Function: The parameters in the tissue IRF's and the parameters in the PTAC model were estimated simultaneously using the weighted nonlinear least-squares (WNLS) method. The total number of parameters to be estimated in $m$-ROI's $(m>1)$ is $5 m$, as there are five parameters $\left(k_{1}^{*}-k_{4}^{*}\right.$ and CBV) in the FDG model. Thus, the total number of parameters to be estimated in simultaneous fitting is $5 m+\mathcal{P}$, where $\mathcal{P}$ is the number of parameters to be estimated in PTAC. The value of this number is dependent on whether two blood samples are used in fitting. If two blood samples are used, i.e., $A_{3}$ and $\lambda_{3}$ are known a priori, $\mathcal{P}$ is equal to four, as only $A_{1}, \lambda_{1}, A_{2}$, and $\lambda_{2}$ in PTAC are to be estimated; otherwise, $\mathcal{P}$ should be equal to six, which is the total number of parameters given in (1).

In the simulation, the Levenberg-Marquardt algorithm [19], [20] was used to optimize the following cost function:

$$
\begin{gathered}
\Phi(\theta)=\sum_{i=1}^{m} \sum_{j=1}^{n} w_{i j}\left\{\left[c_{p}^{*}(t) \otimes h_{i}\left(k_{1}^{*}, k_{2}^{*}, k_{3}^{*}, k_{4}^{*}, \mathrm{CBV} ; t\right)\right]\right. \\
\left.\otimes \delta\left(t-t_{j}\right)-c_{T_{i}}^{*}\left(t_{j}\right)\right\}^{2}
\end{gathered}
$$

where $c_{p}^{*}(t)$ is the PTAC function (1) with $\mathcal{P}$ (either four or six) parameters to be estimated; $h_{i}\left(k_{1}^{*}, k_{2}^{*}, k_{3}^{*}, k_{4}^{*}, \mathrm{CBV} ; t\right)$ is the impulse response of the $i$ th ROI, in which $k_{1}^{*}-k_{4}^{*}$ are the rate constant parameters and $\mathrm{CBV}$ is the cerebral blood volume; $\delta(t)$ is a Dirac-delta function; $n$ is the number of samples for each TTAC; $c_{T_{i}}$ is the $i$ th tissue response curve, that is, the $i$ th TTAC function; and $\theta$ is a parameter vector containing the $5 m+\mathcal{P}$ parameters. For the WNLS method, the weight during the simulation is the inverse of the error variance. Therefore, the weight $w_{i j}$, which is equal to the inverse of the error variance $\left(1 / \sigma_{i j}^{2}\right)$ is used to obtain the smallest variance of estimates. The estimation method, which uses $\Phi(\theta)$ as the cost function, is referred to as the new method in the following discussion.

\section{Statistical Criteria}

Various statistical values, such as mean, standard deviation (SD), coefficient of variation (CV), and bias were calculated from the 100 simulation runs for each noise level. Only one run was done for noise-free data. The coefficient of variation of a parameter estimate is calculated from

$$
\mathrm{CV}_{\bar{P}}=\frac{\mathrm{SD}_{\bar{P}}}{|\bar{P}|} \times 100 \%
$$

where $\bar{P}$ is the parameter estimated over 100 runs and $\mathrm{SD}_{\bar{P}}$ is the standard deviation of $\bar{P}$, and the bias is calculated in the following way:

$$
\text { Bias }=\left|\frac{P^{\text {true }}-\bar{P}}{P^{\text {true }}}\right| \times 100 \%
$$

where $P^{\text {true }}$ is the true parameter value of $P$, and $\bar{P}$ is the mean value obtained from the proposed method. The above statistical values were mainly used as the criteria to evaluate the performance of the proposed method.

\section{RESUlTS AND DisCUSSION}

\section{A. Recovery of the Input Function}

Table II shows the statistical results of PTAC function parameters with a different number of ROI's used, in which (a) corresponds to the results obtained from noise-free data ( $\alpha=0.0$ ) and (b)-(f) correspond to the results obtained from noisy data under different noise levels. In this table, the mean values and the biases of the parameter estimates are given. For each entry showing the number of ROI's used, there are two rows of results, in which the top rows (designated by i) are the results obtained without the use of blood samples, while the results shown at the bottom rows (designated by ii) are obtained with the use of blood samples. Note that the values and biases of $A_{3}$ and $\lambda_{3}$ are equal to their true values and zero, for all rows designated by ii (i.e., with blood samples) for a different number of ROI's used, since the two blood samples are kept constant, which in turn, have fixed the values of these two parameters. The residual sum of squares (RSS) and the area under the estimated curves (AUC's) are also tabulated in the last two columns of the same table.

It would be useful to know how many ROI's are adequate for accurate parameter estimates with reasonable computational complexity. From Table II, it is clearly seen that the estimation results of the PTAC function parameters obtained from three and four ROI's are almost equivalent and are generally better than those obtained from two ROI's, by assessing the biases of the parameter estimates to their true values under the same noise level. In other words, two ROI's are already able to provide sufficient information to quantify the PTAC function. As the number of ROI's used increases, the biases of the parameter estimates and RSS are generally decreased, and the calculated AUC's will approach the one covered by true PTAC. This is as expected because more ROI's may provide more information of the input function to be recovered if the additional ROI's have kinetics differences from those being used.

The advantages of using the blood samples are obvious if we analyze the RSS and AUC's given in Table II. The RSS of PTAC's generated with the parameter estimates obtained 
TABLE II

Estimation Results of the Input Function Parameters $A_{1}, \lambda_{1}, A_{2}, \lambda_{2}, A_{3}$, and $\lambda_{3}$ FOR Different Noise Levels. $\alpha$ Defines the Simulated Noise Level. The Mean Values and the Biases Are Calculated from 100 Simulation Runs for Noisy Data and One Run for Noise-Free Data. Note that There Are Two Rows of Results for Using a Different Number of ROI's Under Different Noise Levels. The Top Rows (Designated by i) Are the Results Obtained Without Using the Two Blood Samples, While the Bottom Rows (Designated by ii) Are the Results Obtained with the Two Blood Samples. Area Under the Calculated PTAC's and the Residual Sum of Squares for Each Curve to the True Input Curve Are Also Listed

\begin{tabular}{|c|c|c|c|c|c|c|c|c|c|c|c|c|c|c|c|}
\hline \multirow{3}{*}{\multicolumn{2}{|c|}{$\begin{array}{l}\text { ROIs } \\
\text { used }\end{array}$}} & \multicolumn{12}{|c|}{ Parameter estimates } & \multirow{3}{*}{$\mathrm{RSS}^{*}$} & \multirow{3}{*}{ AUC\# } \\
\hline & & \multicolumn{2}{|l|}{$A_{1}$} & \multicolumn{2}{|c|}{$\lambda_{1}$} & \multicolumn{2}{|c|}{$A_{2}$} & \multicolumn{2}{|c|}{$\lambda_{2}$} & \multicolumn{2}{|c|}{$A_{3}$} & \multicolumn{2}{|c|}{$\lambda_{3}$} & & \\
\hline & & mean & bias & mean & bias & mean & bias & mean & bias & mean & bias & mean & bias & & \\
\hline \multicolumn{2}{|c|}{ true value } & 851.1225 & 0.0 & -4.1339 & 0.0 & 21.8798 & 0.0 & -0.1191 & 0.0 & 20.8113 & 0.0 & -0.0104 & 0.0 & 0.00 & 1649.81 \\
\hline \multicolumn{16}{|c|}{ (a): 1 run with noise level $\alpha=0.0$} \\
\hline \multirow{2}{*}{2 -ROIs } & i & 851.1225 & 0.0 & -4.1339 & 0.0 & 21.8798 & 0.0 & -0.1191 & 0.0 & 20.8113 & 0.0 & -0.0104 & 0.0 & 0.00 & 1649.81 \\
\hline & ii & 851.1225 & 0.0 & -4.1339 & 0.0 & 21.8798 & 0.0 & -0.1191 & 0.0 & 20.8113 & 0.0 & -0.0104 & 0.0 & 0.00 & 1649.81 \\
\hline \multirow{2}{*}{ 3-ROIs } & i & 851.1225 & 0.0 & -4.1339 & 0.0 & 21.8798 & 0.0 & -0.1191 & 0.0 & 20.8113 & 0.0 & -0.0104 & 0.0 & 0.00 & 1649.81 \\
\hline & ii & 851.1225 & 0.0 & -4.1339 & 0.0 & 21.8798 & 0.0 & -0.1191 & 0.0 & 20.8113 & 0.0 & -0.0104 & 0.0 & 0.00 & 1649.81 \\
\hline \multirow{2}{*}{ 4-ROIs } & i & 851.1225 & 0.0 & -4.1339 & 0.0 & 21.8798 & 0.0 & -0.1191 & 0.0 & 20.8113 & 0.0 & -0.0104 & 0.0 & 0.00 & 1649.81 \\
\hline & ii & 851.1225 & 0.0 & -4.1339 & 0.0 & 21.8798 & 0.0 & -0.1191 & 0.0 & 20.8113 & 0.0 & -0.0104 & 0.0 & 0.00 & 1649.81 \\
\hline \multicolumn{16}{|c|}{ (b): 100 runs with noise level $\alpha=0.1$} \\
\hline \multirow{2}{*}{ 2-ROIs } & i & 907.7383 & 6.7 & -4.2176 & 2.0 & 27.7798 & 26.7 & -0.1791 & 50.4 & 21.6935 & 4.2 & -0.0191 & 83.7 & 279.43 & 1215.41 \\
\hline & ii & 785.5303 & 7.7 & -4.2311 & 2.4 & 21.6236 & 1.2 & -0.1266 & 6.3 & 20.8113 & 0.0 & -0.0104 & 0.0 & 126.95 & 1631.27 \\
\hline \multirow{2}{*}{ 3-ROIs } & i & 1092.9739 & 28.4 & -4.1887 & 1.3 & 27.2268 & 24.4 & -0.1812 & 52.1 & 21.5089 & 3.4 & -0.0188 & 80.8 & 1182.18 & 1225.14 \\
\hline & ii & 880.3888 & 3.4 & -4.2105 & 1.9 & 21.8590 & 1.0 & -0.1225 & 2.9 & 20.8113 & 0.0 & -0.0104 & 0.0 & 3.28 & 1644.58 \\
\hline 4-BOIs & $\mathrm{i}$ & $8 \overline{99.2629}$ & 5.7 & -4.1977 & 1.5 & 26.0864 & 19.2 & -0.1210 & 1.6 & 20.8930 & 0.4 & -0.0121 & 16.3 & 163.29 & 1577.91 \\
\hline & ii & 877.6562 & 3.1 & -4.1960 & 1.5 & 21.9050 & 6.1 & -0.1213 & 1.8 & 20.8113 & 0.0 & -0.0104 & 0.0 & 2.50 & 1646.87 \\
\hline & & & & & 100 & ins with I & ise ley & $\alpha \alpha=0.5$ & & & & & & & \\
\hline $2-B$ & i & 1097.8081 & 29.0 & -4.2224 & 2.1 & 26.2661 & 20.0 & -0.1812 & 52.1 & 21.1459 & 1.6 & -0.0175 & 68.3 & 985.04 & 1255.67 \\
\hline $2-\mathrm{F}$ & ii & 939.0012 & 10.3 & -4.3062 & 4.2 & 22.2780 & 1.8 & -0.1214 & 1.9 & 20.8113 & 0.0 & -0.0104 & 0.0 & 29.41 & 1650.76 \\
\hline 3-BOIs & $\bar{i}$ & 1061.2721 & 24.7 & -4.2257 & 2.2 & 26.6108 & 21.6 & -0.1803 & 51.4 & 20.8849 & 0.4 & -0.0171 & 64.4 & 756.99 & 1260.21 \\
\hline 3-kU15 & ii & 909.3292 & 6.8 & -4.2466 & 2.7 & 22.2706 & 1.8 & -0.1194 & 0.3 & 20.8113 & 0.0 & -0.0104 & 0.0 & 14.61 & 1653.41 \\
\hline 4 -ROIs & i & 919.7122 & 8.1 & -4.2227 & 2.1 & 25.0314 & 14.4 & -0.1157 & 2.9 & 20.3098 & 2.4 & -0.0130 & 25.0 & 108.00 & 1491.19 \\
\hline 4-kUls & ii & 910.4514 & 7.0 & -4.2193 & 2.1 & 22.1317 & 1.2 & -0.1204 & 1.1 & 20.8113 & 0.0 & -0.0104 & 0.0 & 19.64 & 1651.40 \\
\hline & & & & & 100 & ins with r & ise le & $\alpha=1.0$ & & & & & & & \\
\hline 2-BOIs & i & 1030.1894 & 21.0 & -3.9574 & 4.3 & 26.8702 & 22.8 & -0.1840 & 54.5 & 21.4619 & 3.1 & -0.0180 & 73.1 & 1365.01 & 1254.42 \\
\hline & ii & 926.4015 & 8.8 & -4.4765 & 8.3 & 21.9403 & 0.3 & -0.1239 & 4.0 & 20.8113 & 0.0 & -0.0104 & 0.0 & 44.86 & 1640.38 \\
\hline 3-ROIs & $\mathrm{i}$ & 1006.9439 & 18.3 & -4.3819 & 6.0 & $28 . \overline{3321}$ & 29.5 & -0.1086 & 8.8 & 20.1719 & 3.1 & -0.0132 & 26.9 & 446.14 & 1516.92 \\
\hline 3-RUUis & ii & 981.8803 & 15.4 & -4.4090 & 6.7 & 21.9760 & 0.4 & -0.1191 & 0.0 & 20.8113 & 0.0 & -0.0104 & 0.0 & 55.74 & 1651.94 \\
\hline 4. $\mathrm{ROIs}$ & $i$ & 964.4576 & 13.3 & -4.3179 & 4.5 & $26.12 \overline{2} 4$ & 19.4 & -0.1068 & 10.3 & 19.9775 & 4.0 & -0.0127 & 22.1 & 191.95 & $\overline{1516.01}$ \\
\hline $4-\mathrm{hU}$ Us & ii & 913.0922 & 7.3 & -4.3578 & 5.4 & 22.1739 & 1.3 & -0.1192 & 0.1 & 20.8113 & 0.0 & -0.0104 & 0.0 & 14.19 & 1650.86 \\
\hline & & & & & 100 & ins with $r$ & ise ley & $\alpha=2.0$ & & & & & & & \\
\hline $2 \mathrm{~B}$ & $i$ & 1092.2661 & 28.3 & -4.9384 & 19.5 & 27.4472 & 25.4 & -0.1880 & 57.9 & 21.0809 & 1.3 & -0.0177 & 70.2 & 226.72 & 1229.58 \\
\hline $2-\mathrm{T}$ & ii & 911.0770 & 7.0 & -4.5291 & 9.6 & 22.2017 & 1.5 & -0.1257 & 5.5 & 20.8113 & 0.0 & -0.0104 & 0.0 & 73.10 & 1638.16 \\
\hline & i & 962.0124 & 13.0 & -4.1962 & 1.5 & 24.1430 & 10.3 & -0.1038 & 12.8 & 19.8406 & 4.7 & -0.0125 & 20.2 & 177.55 & 1509.83 \\
\hline & ii & 854.0221 & 0.4 & -4.2433 & 2.6 & 22.4233 & 2.5 & -0.1197 & 0.5 & 20.8113 & 0.0 & -0.0104 & 0.0 & 8.74 & 1651.19 \\
\hline 4 & i & 926.5917 & 8.9 & -4.2344 & 2.4 & 25.9773 & 18.7 & -0.1055 & 11.4 & 19.7864 & 4.9 & -0.0132 & 26.9 & 154.18 & 1478.55 \\
\hline IS & ii & 850.9847 & 0.0 & -4.2126 & 1.9 & 22.3236 & 2.0 & -0.1217 & 2.2 & 20.8113 & 0.0 & -0.0104 & 0.0 & 5.31 & 1647.76 \\
\hline & & & & & 100 & ns with $n$ & ise ley & $\alpha \alpha=4.0$ & & & & & & & \\
\hline 2- $\mathrm{BO} \mathrm{s}$ & i & 1343.7089 & 57.9 & -4.6371 & 12.2 & 25.7558 & 17.7 & -0.1830 & 53.7 & 20.9690 & 0.8 & -0.0172 & 65.4 & 1523.59 & 1257.52 \\
\hline $2-\mathrm{RO}$ ls & ii & 1182.1540 & 38.9 & -4.9993 & 20.9 & 21.8519 & 0.1 & -0.1260 & 5.8 & 20.8113 & 0.0 & -0.0104 & 0.0 & 249.26 & 1638.81 \\
\hline 3-ROTs & i & $10 \overline{91.9457}$ & 28.3 & -4.3527 & 5.3 & 23.6479 & 8.1 & -0.1020 & 14.4 & 19.9308 & 4.2 & -0.0124 & 19.2 & 437.63 & 1523.81 \\
\hline & ii & 923.2316 & 8.5 & -4.4767 & 8.3 & 22.1959 & 1.4 & -0.1190 & 0.1 & 20.8113 & 0.0 & -0.0104 & 0.0 & 38.68 & 1649.60 \\
\hline 4-ROIs & i & 941.2364 & 10.6 & -4.2809 & 3.6 & 24.9430 & 14.0 & -0.1044 & 12.3 & 19.7817 & 4.9 & -0.0132 & 26.9 & 103.73 & 1471.00 \\
\hline & ii & 869.2379 & 2.1 & -4.3710 & 5.7 & 22.0382 & 0.7 & -0.1164 & 2.3 & 20.8113 & 0.0 & -0.0104 & 0.0 & 42.30 & 1651.64 \\
\hline
\end{tabular}

${ }^{*}$ RSS $=$ Residual sum of squares.

\# AUC $=$ Area under the calculated PTAC from 0 to 120 minutes.

without using the blood samples in estimation are much larger than those with them. The AUC's, however, are much less than those calculated from PTAC's, whose parameters are estimated with blood samples. In addition, the quality of the parameter estimates is poor in terms of the biases if the blood samples are not used in the estimation process. It appears that $\lambda_{3}$ deviates very much from their true values, though the scaling parameter $A_{3}$ can be estimated very accurately.

On the other hand, the recovered input curves generated from the parameter estimates obtained with the use of blood samples can closely resemble the true input curve. The RSS and biases are generally much better than those estimated without the use of blood samples, and the calculated AUC's are very close to the one covered by the true input function. The true input function and the fitted PTAC's estimated with a different number of ROI's at the highest noise level $(\alpha=4.0)$ are plotted in Fig. 4. It is not difficult to see that the estimated input curve is underestimated after $20 \mathrm{~min}$ [Fig. 4(a)] and the peak region is overestimated [Fig. 4(b)] if the blood samples are not used during estimation. As a result, the area covered is underestimated by $9-25 \%$ on the average. However, the fitted PTAC's (with the use of blood samples during estimation) agree well with the true input function in terms of their shapes and the areas covered.

\section{B. Estimation of $k_{1}^{*}-k_{4}^{*}, C B V$, and $K$}

Tables III and IV summarize the results of the IRF's parameters estimated using two, three, and four ROI's, with and without the blood samples in the estimation process. Tables III(a) and IV(a) correspond to the simulation results of noise-free data, while Tables III(b)-(f) and IV(b)-(f) correspond to the simulation results obtained from data with different noise levels by using two, three, and four ROI's, respectively, in the estimation of $k_{1}^{*}-k_{4}^{*}, \mathrm{CBV}$, and the combined parameter $K$ in grey matter and white matter. It can be seen that two ROI's have already been able to provide 


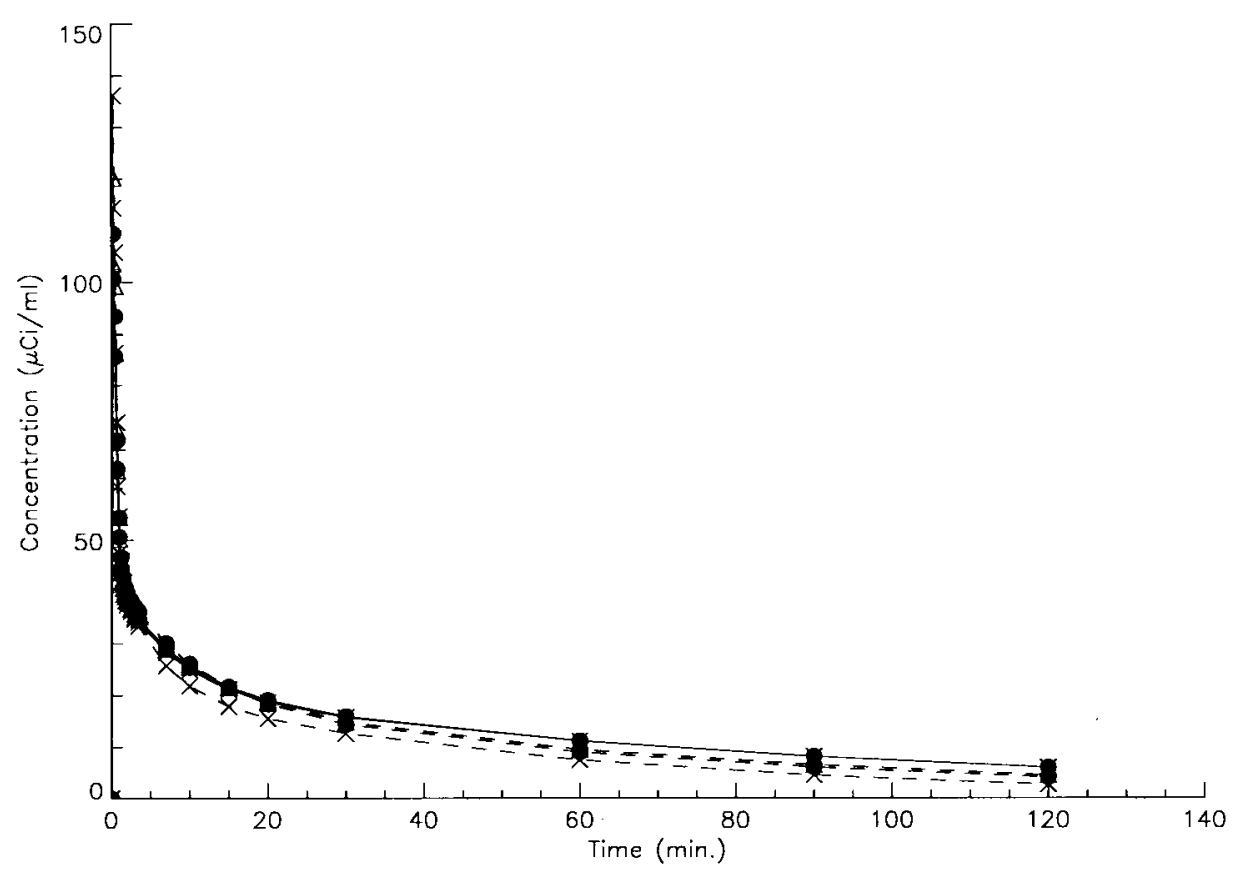

(a)

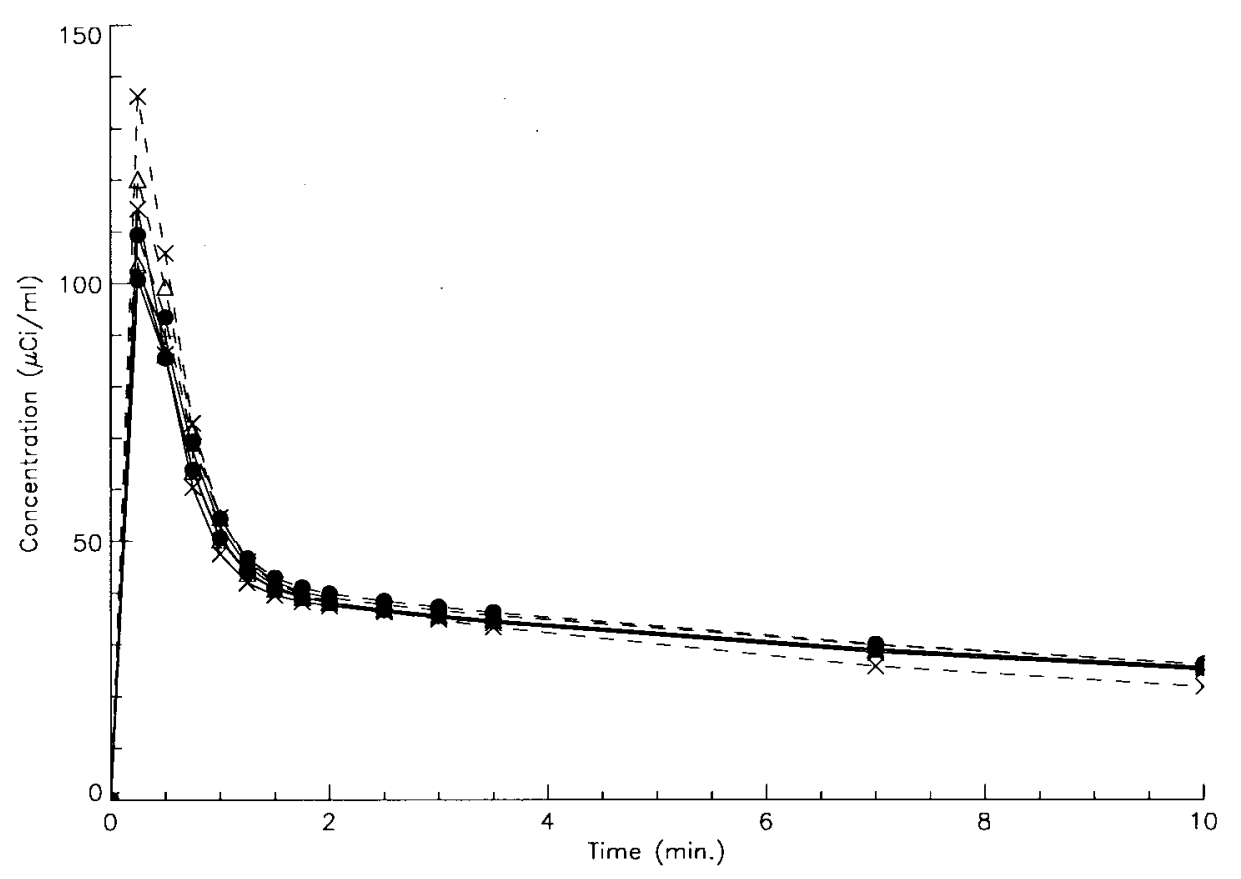

(b)

Fig. 4. (a) Graph shows the generated input function and the fitted PTAC's obtained from using a different number of ROI's in simulation (noise level $\alpha=4.0$ ) and (b) graph shows the expanded peak region from 0-10 min. In all of the graphs, solid lines with symbols $\times, \Delta$, and $\bullet$ are the results obtained from using two venous samples with two, three, and four ROI's, respectively, while dashed lines with symbols $\times, \Delta$, and $\bullet$ are the results obtained from using image information only, i.e., without using the two venous samples for parameter estimation. A solid line with symbol + is the generated input function.

sufficient information to quantify the parameters in IRF's and PTAC. In general, additional ROI's can provide improved parameter estimation accuracy. However, there is no significant improvement of parameter estimation accuracy if more than three ROI's are used, unless the kinetics in the fourth region are very different from those in the three regions being used.
It would be of interest to see the improvement in parameter estimation accuracy if late blood samples are used to help recovery of the input function and the estimation of $k_{1}^{*}-k_{4}^{*}$, CBV in IRF's. Without using blood samples in the estimation process, there are very large fluctuations in parameter estimates of IRF's. It can been seen from Tables III and IV that the 
TABLE III

Estimates of Rate Constants $k_{1}^{*}-k_{4}^{*}$, the CBV, and the Combined Parameter $K$ in Grey Matter for Different Noise Levels, Estimated with and Without Using the Information of the Two Blood Samples. $\alpha$ Corresponds to the Proportionality Constant that Defines the Simulated Noise Levels. The Mean Values $\bar{k}_{1}^{*}-\bar{k}_{4}^{*}, \overline{\mathrm{CBV}}$, and $\bar{K}$ Are Calculated from 100 Simulation Runs

\begin{tabular}{|c|c|c|c|c|c|c|c|c|c|c|c|c|}
\hline \multirow{3}{*}{$\begin{array}{l}\text { No. of } \\
\text { ROIs } \\
\text { used }\end{array}$} & \multicolumn{12}{|c|}{ Parameter estimates } \\
\hline & \multicolumn{6}{|c|}{ Without blood samples } & \multicolumn{6}{|c|}{ With blood samples } \\
\hline & $k_{1}^{*}$ & $k_{2}^{*}$ & $\bar{k}_{3}^{*}$ & $k_{4}^{*}$ & $\overline{C B V}$ & $K$ & $k_{1}^{*}$ & $k_{2}^{*}$ & $k_{3}^{*}$ & $k_{4}^{*}$ & $\overline{C B V}$ & $\bar{K}$ \\
\hline true value & 0.0410 & 0.1190 & 0.0640 & 0.0075 & 5.80 & 0.0143 & 0.0410 & 0.1190 & 0.0640 & 0.0075 & 5.80 & 0.0143 \\
\hline \multicolumn{13}{|c|}{ (a): 1 run with noise level $\alpha=0.0$} \\
\hline 2-ROIs & 0.0410 & 0.1190 & 0.0640 & 0.0075 & 5.80 & 0.0143 & 0.0410 & 0.1190 & 0.0640 & 0.0075 & 5.80 & 0.0143 \\
\hline 3-ROIs & 0.0410 & 0.1190 & 0.0640 & 0.0075 & 5.80 & 0.0143 & 0.0410 & 0.1190 & 0.0640 & 0.0075 & 5.80 & 0.0143 \\
\hline 4-ROIs & 0.0410 & 0.1190 & 0.0640 & 0.0075 & 5.80 & 0.0143 & 0.0410 & 0.1190 & 0.0640 & 0.0075 & 5.80 & 0.0143 \\
\hline \multicolumn{13}{|c|}{ (b): 100 runs with noise level $\alpha=0.1$} \\
\hline 2 -ROIs & 0.0361 & 0.0801 & 0.0639 & 0.0016 & 5.55 & 0.0159 & 0.0405 & 0.1115 & 0.0616 & 0.0075 & 6.54 & 0.0144 \\
\hline 3-ROIs & 0.0367 & 0.0828 & 0.0638 & 0.0020 & 4.73 & 0.0159 & 0.0405 & 0.1152 & 0.0628 & 0.0075 & 5.93 & 0.0143 \\
\hline 4-ROIs & 0.0369 & 0.1106 & 0.0708 & 0.0066 & 5.65 & 0.0144 & 0.0405 & 0.1158 & 0.0630 & 0.0075 & 5.90 & 0.0143 \\
\hline \multicolumn{13}{|c|}{ (c): 100 runs with noise level $\alpha=0.5$} \\
\hline 2-ROIs & 0.0374 & 0.0831 & 0.0588 & 0.0020 & 4.81 & 0.0154 & 0.0401 & 0.1155 & 0.0629 & 0.0074 & 5.97 & 0.0142 \\
\hline 3-ROIs & 0.0377 & 0.0838 & 0.0574 & 0.0019 & 4.94 & 0.0153 & 0.0402 & 0.1171 & 0.0637 & 0.0074 & 5.90 & 0.0142 \\
\hline 4-ROIs & 0.0389 & 0.1155 & 0.0722 & 0.0059 & 5.72 & 0.0148 & 0.0403 & 0.1165 & 0.0631 & 0.0074 & 5.79 & 0.0142 \\
\hline \multicolumn{13}{|c|}{ (d): 100 runs with noise level $\alpha=1.0$} \\
\hline 2-ROIs & 0.0380 & 0.0921 & 0.0637 & 0.0018 & 5.20 & 0.0153 & 0.0407 & 0.1195 & 0.0636 & 0.0074 & 6.42 & 0.0142 \\
\hline 3-ROIs & 0.0364 & 0.1288 & 0.0819 & 0.0058 & 5.41 & 0.0143 & 0.0409 & 0.1224 & 0.0651 & 0.0075 & 5.98 & 0.0142 \\
\hline 4-ROIs & 0.0394 & 0.1441 & 0.0852 & 0.0058 & 5.53 & 0.0146 & 0.0408 & 0.1232 & 0.0657 & 0.0075 & 6.06 & 0.0143 \\
\hline \multicolumn{13}{|c|}{ (e): 100 runs with noise level $\alpha=2.0$} \\
\hline 2-ROIs & 0.0382 & 0.1000 & 0.0653 & 0.0018 & 5.13 & 0.0153 & 0.0403 & 0.1192 & 0.0636 & 0.0073 & 6.67 & 0.0142 \\
\hline 3-ROIs & 0.0401 & 0.1357 & 0.0740 & 0.0056 & 5.66 & 0.0145 & 0.0403 & 0.1217 & 0.0656 & 0.0074 & 6.24 & 0.0142 \\
\hline 4-ROIs & 0.0390 & 0.1370 & 0.0792 & 0.0051 & 5.70 & 0.0145 & 0.0403 & 0.1198 & 0.0650 & 0.0074 & 6.22 & 0.0142 \\
\hline \multicolumn{13}{|c|}{ (f): 100 runs with noise level $\alpha=4.0$} \\
\hline 2-ROIs & 0.0392 & 0.1029 & 0.0656 & 0.0023 & 4.81 & 0.0152 & 0.0414 & 0.1304 & 0.0638 & 0.0071 & 6.44 & 0.0140 \\
\hline 3-ROIs & 0.0404 & 0.1426 & 0.0738 & 0.0055 & 5.42 & 0.0142 & 0.0408 & 0.1290 & 0.0660 & 0.0072 & 6.37 & 0.0141 \\
\hline 4-ROIs & 0.0399 & 0.1436 & 0.0792 & 0.0046 & 5.55 & 0.0145 & 0.0410 & 0.1284 & 0.0657 & 0.0072 & 6.35 & 0.0141 \\
\hline
\end{tabular}

forward-transport rate constant $k_{1}^{*}$ and the dephosphorylation rate constant $k_{4}^{*}$ are significantly underestimated $(2-15 \%$ for $k_{1}^{*}$ and $10-80 \%$ for $k_{4}^{*}$ ), while there are very large deviations of the reverse-transport rate constant $k_{2}^{*}$ (maximum $\pm 25 \%$ ) and the phosphorylation rate constant $k_{3}^{*}$ (maximum $\pm 20 \%$ ) from their true values. However, their parameter estimates are very stable if blood samples are used during the estimation. For the $\mathrm{CBV}$, the fluctuations of parameter estimates are moderate (about $10 \%$ on the average), which reveal that the parameter estimates are at an acceptable accuracy level.

Figs. 5 and 6 plot the CV's as a function of noise level for all of the parameters in IRF's of grey matter and white matter, respectively. By investigating the CV's, we can observe that the CV's of some of the parameter estimates are very large even if blood samples are used in the estimation process. Examples include the estimation of $k_{2}^{*}$ and $k_{3}^{*}$. As expected, the forward-transport rate constant $k_{1}^{*}$ can be estimated very accurately because it is the most sensitive parameter in the FDG model. The dephosphorylation rate constant $k_{4}^{*}$, however, is the poorest among all parameters in IRF. The improvement in parameter estimation with the use of blood samples is also revealed in these figures. The CV's of parameter estimates obtained without the use of blood samples are generally higher than those obtained with them. This justifies the use of blood samples to help the estimation of parameters in IRF's and the recovery of the input function without introducing any additional burden, but it greatly improves the quality of identification in the present study.

Note that one of our major goals in modeling is to estimate $K$ or, equivalently, the LCMRGlc. The statistical results given in Tables III and IV demonstrate that $K$ can be estimated very reliably with the new method. It is clear that if late blood samples are used in the estimation, the new method can provide an unbiased estimation of $K$. Considerable overestimation of $K$ (up to 11 and $5 \%$ on the average) can be observed if late blood samples have not been used in the estimation. This may be due to very large variations in rate constants $k_{2}^{*}$ and $k_{3}^{*}$ and significant underestimation of $k_{1}^{*}$ and $k_{4}^{*}$. Figs. 5(f) and 6(f) show the CV's of estimated values of $K$ in grey matter and white matter, respectively. It can be seen that the CV's of $K$ obtained without the blood samples are generally larger than those with them. This is as expected since uncertainties in rate constants are much smaller and their estimates are more reliable if the blood samples are used during the estimation. As a result, $K$ can be estimated very accurately and the corresponding CV's are also smaller.

As discussed previously, if output data obtained from a single ROI alone are used, we are able to identify neither the FDG model parameters nor the input function parameters. From the above results, we can see that when two ROI's 
TABLE IV

Estimates of the Rate Constants $k_{1}^{*}-k_{4}^{*}$, the CBV, and the Combined Parameter $K$ in White Matter in Different Noise Levels, Estimated with and Without Using the Information of the Two Blood Samples. $\alpha$ Corresponds to the Proportionality Constant that Defines the Simulated Noise Levels. The Mean Values $\bar{k}_{1}^{*}-\bar{k}_{4}^{*}, \overline{\mathrm{CBV}}$, and $\bar{K}$ Are Calculated from 100 Simulation Runs

\begin{tabular}{|c|c|c|c|c|c|c|c|c|c|c|c|c|}
\hline \multirow{3}{*}{$\begin{array}{c}\text { No. of } \\
\text { ROIs } \\
\text { used }\end{array}$} & \multicolumn{12}{|c|}{ Parameter estimates } \\
\hline & \multicolumn{6}{|c|}{ Without blood samples } & \multicolumn{6}{|c|}{ With blood samples } \\
\hline & $k_{1}^{*}$ & $\bar{k}_{2}^{*}$ & $k_{3}^{*}$ & $\bar{k}_{4}^{*}$ & $\overline{C B V}$ & $\bar{K}$ & $\overline{k_{1}^{*}}$ & $k_{2}^{*}$ & $\overline{k_{3}^{*}}$ & $k_{4}^{*}$ & $C B V$ & $\bar{K}$ \\
\hline true value & 0.0230 & 0.0730 & 0.0340 & $0.0098^{\circ}$ & 2.50 & 0.0073 & 0.0230 & 0.0730 & 0.0340 & 0.0098 & 2.50 & 0.0073 \\
\hline \multicolumn{13}{|c|}{ (a): 1 run with noise level $\alpha=0.0$} \\
\hline 2-ROIs & 0.0230 & 0.0730 & 0.0340 & 0.0098 & 2.50 & 0.0073 & 0.0230 & 0.0730 & 0.0340 & 0.0098 & 2.50 & 0.0073 \\
\hline 3-ROIs & 0.0230 & 0.0730 & 0.0340 & 0.0098 & 2.50 & 0.0073 & 0.0230 & 0.0730 & 0.0340 & 0.0098 & 2.50 & 0.0073 \\
\hline 4-ROIs & 0.0230 & 0.0730 & 0.0340 & 0.0098 & 2.50 & 0.0073 & 0.0230 & 0.0730 & 0.0340 & 0.0098 & 2.50 & 0.0073 \\
\hline \multicolumn{13}{|c|}{ (b): 100 runs with noise level $\alpha=0.1$} \\
\hline 2-ROIs & 0.0208 & 0.0479 & 0.0312 & 0.0022 & 2.37 & 0.0080 & 0.0233 & 0.0720 & 0.0325 & 0.0094 & 2.80 & 0.0072 \\
\hline 3 -ROIs & 0.0254 & 0.0573 & 0.0434 & 0.0018 & 3.57 & 0.0079 & 0.0230 & 0.0720 & 0.0332 & 0.0096 & 2.53 & 0.0072 \\
\hline 4-ROIs & 0.0210 & 0.0671 & 0.0374 & 0.0091 & 2.43 & 0.0075 & 0.0229 & 0.0722 & 0.0334 & 0.0096 & 2.52 & 0.0072 \\
\hline \multicolumn{13}{|c|}{ (c): 100 runs with noise level $\alpha=0.5$} \\
\hline 2-ROIs & 0.0213 & 0.0496 & 0.0287 & 0.0024 & 2.05 & 0.0077 & 0.0228 & 0.0725 & 0.0333 & 0.0093 & 2.56 & 0.0071 \\
\hline 3-ROIs & 0.0214 & 0.0495 & 0.0274 & 0.0020 & 2.10 & 0.0075 & 0.0227 & 0.0728 & 0.0338 & 0.0094 & 2.54 & 0.0072 \\
\hline 4-ROIs & 0.0221 & 0.0702 & 0.0385 & 0.0083 & 2.47 & 0.0077 & 0.0228 & 0.0726 & 0.0335 & 0.0094 & 2.48 & 0.0072 \\
\hline \multicolumn{13}{|c|}{ (d): 100 runs with noise level $\alpha=1.0$} \\
\hline 2-ROIs & 0.0214 & 0.0518 & 0.0306 & 0.0025 & 2.22 & 0.0077 & 0.0232 & 0.0743 & 0.0335 & 0.0092 & 2.75 & 0.0071 \\
\hline 3-ROIs & 0.0205 & 0.0749 & 0.0438 & 0.0087 & 2.32 & 0.0076 & 0.0229 & 0.0737 & 0.0340 & 0.0095 & 2.57 & 0.0072 \\
\hline 4-ROIs & 0.0217 & 0.0773 & 0.0438 & 0.0089 & 2.38 & 0.0078 & 0.0229 & 0.0741 & 0.0343 & 0.0096 & 2.60 & 0.0072 \\
\hline \multicolumn{13}{|c|}{ (e): 100 runs with noise level $\alpha=2.0$} \\
\hline 2-ROIs & 0.0212 & 0.0526 & 0.0313 & 0.0026 & 2.17 & 0.0077 & 0.0231 & 0.0759 & 0.0345 & 0.0091 & 2.82 & 0.0071 \\
\hline 3-ROIs & 0.0219 & 0.0741 & 0.0391 & 0.0077 & 2.41 & 0.0075 & 0.0228 & 0.0755 & 0.0351 & 0.0094 & 2.64 & 0.0072 \\
\hline 4-ROIs & 0.0215 & 0.0774 & 0.0428 & 0.0073 & 2.43 & 0.0076 & 0.0229 & 0.0749 & 0.0347 & 0.0094 & 2.63 & 0.0072 \\
\hline \multicolumn{13}{|c|}{ (f): 100 runs with noise level $\alpha=4.0$} \\
\hline 2-ROIs & 0.0217 & 0.0569 & 0.0330 & 0.0040 & 2.04 & 0.0079 & 0.0234 & 0.0776 & 0.0351 & 0.0093 & 2.72 & 0.0072 \\
\hline 3-ROIs & 0.0226 & 0.0869 & 0.0437 & 0.0086 & 2.31 & 0.0077 & 0.0229 & 0.0763 & 0.0356 & 0.0096 & 2.73 & 0.0072 \\
\hline 4-ROIs & 0.0220 & 0.0837 & 0.0469 & 0.0078 & 2.41 & 0.0079 & 0.0230 & 0.0776 & 0.0362 & 0.0098 & 2.72 & 0.0073 \\
\hline
\end{tabular}

are used, the parameters in the FDG models and the input function are identifiable. However, if the kinetics of these two ROI's are identical, no additional independent information is added. We still have insufficient information to identify the required parameters. In order to estimate the parameters as reliably as possible, the kinetics of these two ROI's should be as different as possible. In this paper, we have used typical TTAC functions in two regions, one corresponding to grey matter and one corresponding to white matter. The kinetics in these two regions are very different, and hence, reasonable estimation can be achieved. We then introduced an additional ROI with its kinetics derived from a tumor. The identifiability has been improved; i.e., a more reliable estimation has been achieved. However, if the third ROI is identical to any one of the two ROI's used, we do not expect to have any improvement in parameter estimation. Instead, it would increase the computational complexity and numerical difficulty, and hence, the parameter estimation accuracy may be reduced. A fourth ROI defined in hemisphere is then added. Although, we have more additional information, the computational complexity and numerical difficulty are increased as well. Therefore, the improvement in accuracy for parameter estimation is not significant, particularly for $K$. From our simulation study, we conclude that using three different ROI's can achieve sufficiently good estimation results in practice.
According to the simulation results, the method proposed is promising for dynamic FDG-PET studies to provide noninvasive quantification of LCMRGlc and the input function. The application to human studies is currently under investigation, and the results will be reported separately.

\section{CONCLUSION}

A noninvasive technique to extract the physiological parameters and the required input function for modeling from the PET dynamic images in conjunction with two or more late blood samples was proposed in this paper. The identifiability of this method was tested by using Monte Carlo simulation. The results show that the proposed method is able to quantify all the required parameters by using the information obtained from two or more ROI's with very different kinetics in the PET dynamic images. When three or more ROI's are used, more reliable parameter estimation can be achieved. However, there is no significant improvement in parameter estimation (for $K$ or, equivalently, LCMRGlc) if the number of ROI's are more than three unless the kinetics in the additional regions are very different from those in the existing three regions. We suggest that three ROI's, with very different kinetics, should be used in practice to achieve reliable estimation and manageable computational complexity. The proposed method can provide very reliable estimation of LCMRGlc, which is the primary 


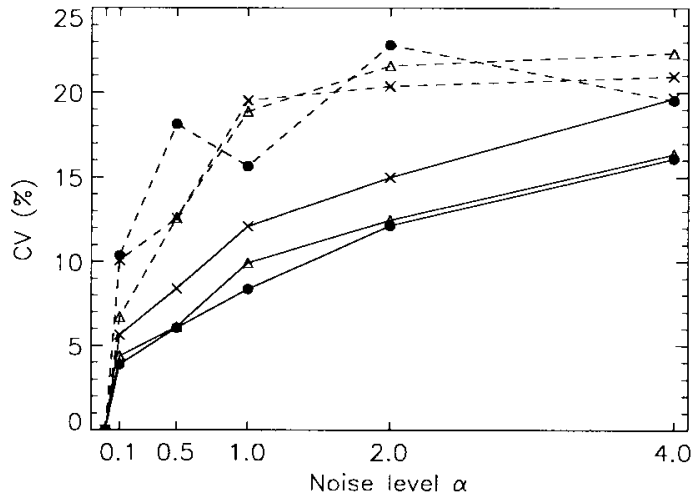

(a)

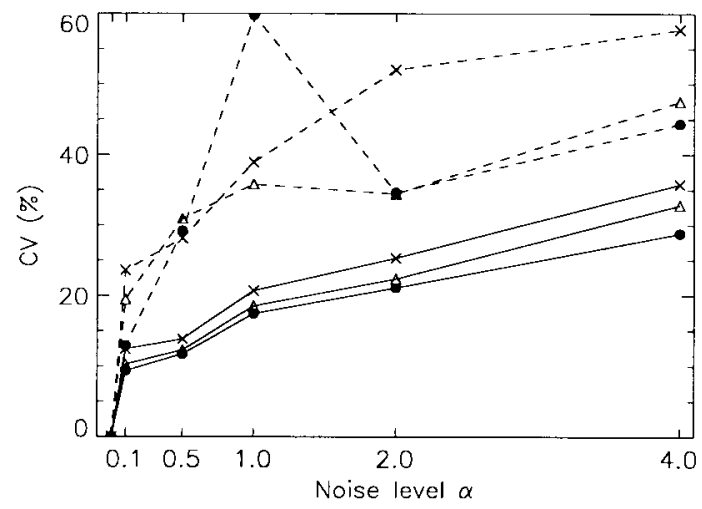

(c)

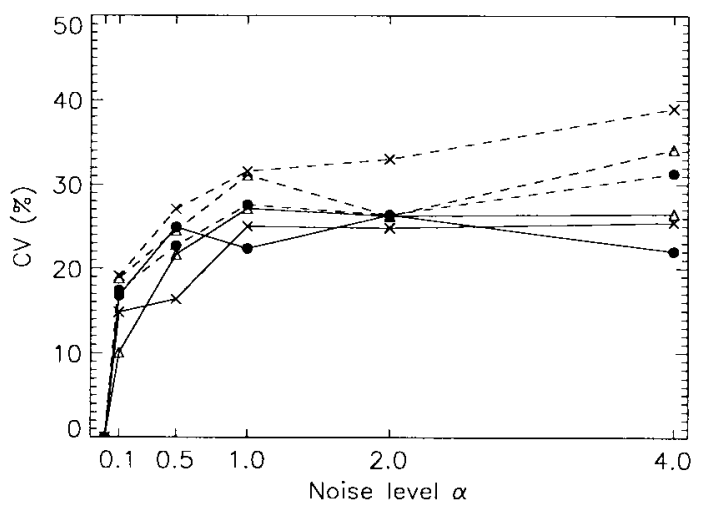

(e)

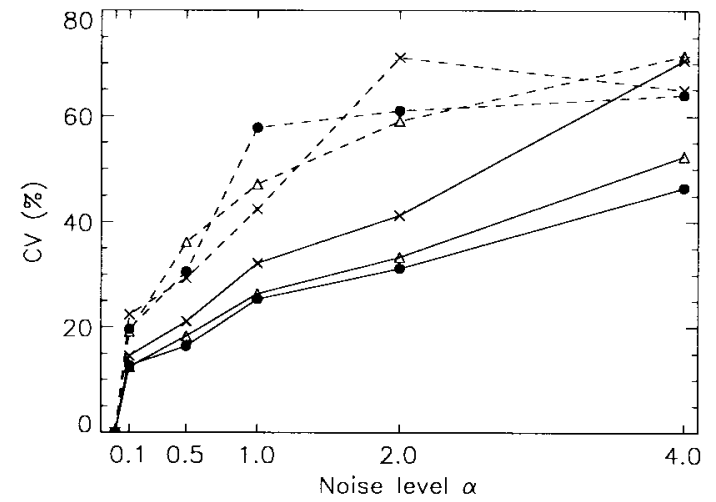

(b)

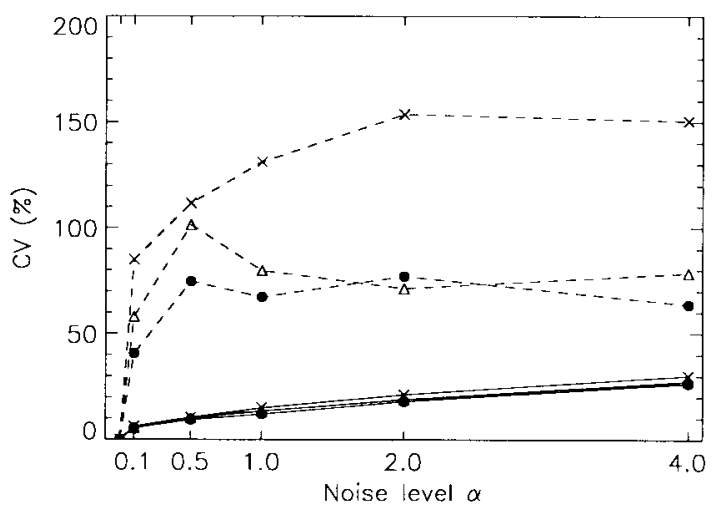

(d)

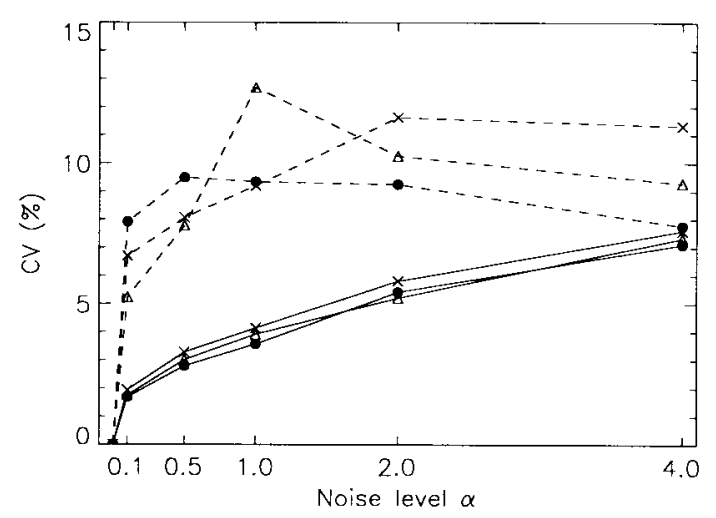

(f)

Fig. 5. Comparison of CV's for (a) $k_{1}^{*}$, (b) $k_{2}^{*}$, (c) $k_{3}^{*}$, (d) $k_{4}^{*}$, (e) CBV, and (f) $K$ in grey matter using two, three, and four ROI's in simulation for different noise levels. In all of the graphs, solid lines with symbols $\times, \Delta$, and $\bullet$ represent the results obtained from using two venous samples with two, three, and four ROI's, respectively, while dashed lines with symbols $\times, \Delta$, and $\bullet$ are the results obtained from using image information only, i.e., without using the two venous samples for parameter estimation.

interest in dynamic FDG studies with PET and may be a more practical alternative to the traditional method, which requires frequent blood sampling during the course of scanning.

\section{ACKNOWLEDGMENT}

The authors are grateful to Dr. S. Meikle and Dr. M. Fulham from the Biomedical and Multimedia Information Technology Group and the Nuclear Medicine and PET Department, Royal
Prince Alfred Hospital, Australia, for their valuable comments on this work.

\section{REFERENCES}

[1] S. C. Huang, M. E. Phelps, E. J. Hoffman, K. Sideris, C. Selin, and D. E. Kuhl, "Noninvasive determination of local cerebral metabolic rate of glucose in man," Amer. J. Physiol., vol. 238, pp. E69-E82, 1980.

[2] M. E. Phelps, S. C. Huang, E. J. Hoffman, C. Selin, L. Sokoloff, and D. E. Kuhl, "Tomographic measurement of local cerebral glucose metabolic 


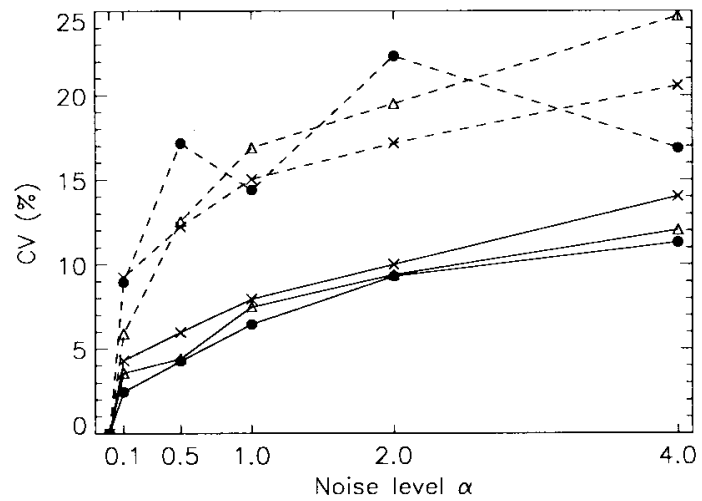

(a)

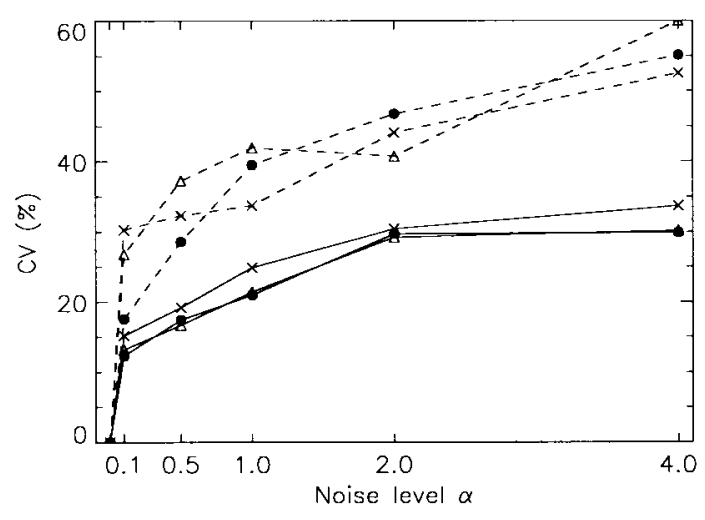

(c)

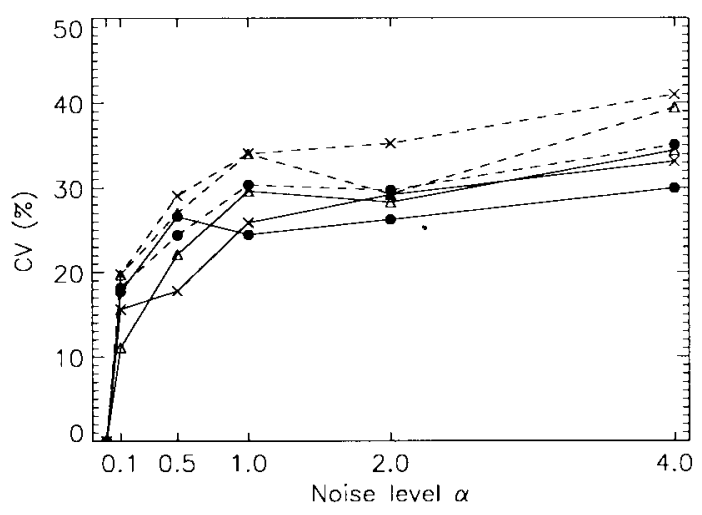

(e)

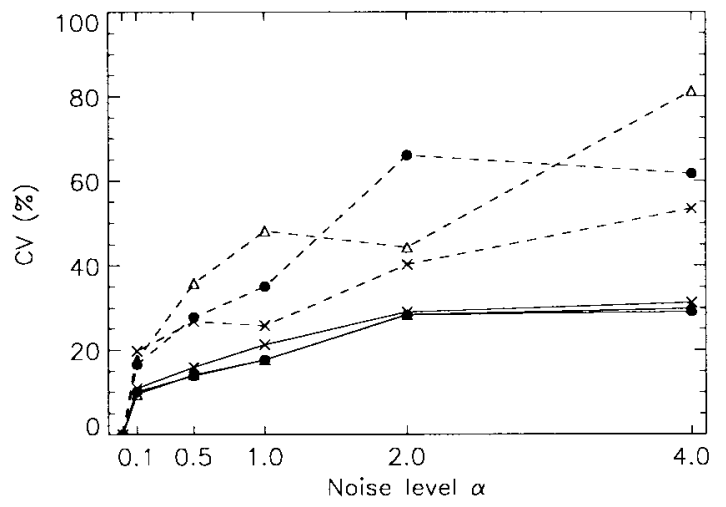

(b)

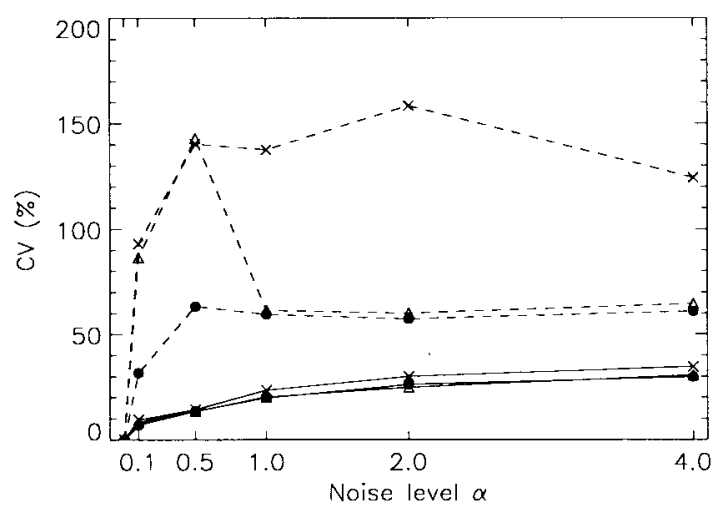

(d)

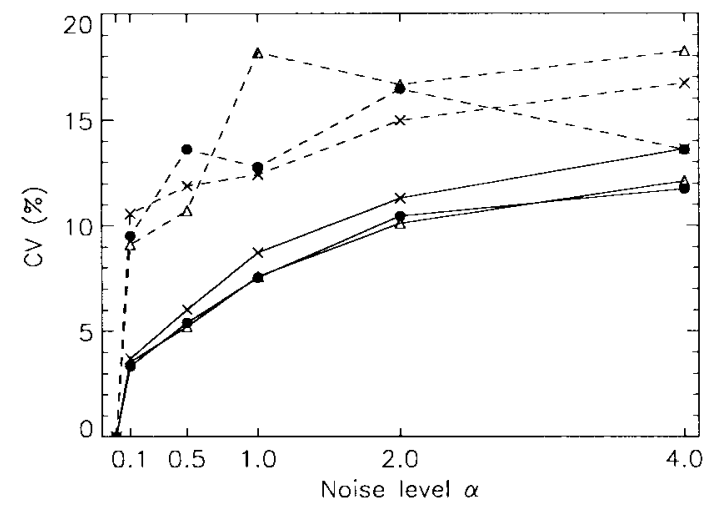

(f)

Fig. 6. Comparison of CV's for (a) $k_{1}^{*}$, (b) $k_{2}^{*}$, (c) $k_{3}^{*}$, (d) $k_{4}^{*}$, (e) CBV, and (f) $K$ in white matter using two, three, and four ROI's in simulation for different noise levels. In all of the graphs, solid lines with symbols $\times, \Delta$, and $\bullet$ represent the results obtained from using two venous samples with two, three, and four ROI's, respectively, while dashed lines with symbols $\times, \Delta$, and $\bullet$ are the results obtained from using image information only, i.e., without using the two venous samples for parameter estimation.

rate in humans with (F-18)2-fluoro-2-deoxy-D-glucose: Validation of method," Ann. Neurol., vol. 6, pp. 371-388, 1979.

[3] J. Correia, "Editorial: A bloody future for clinical PET," J. Nucl. Med., vol. 33, pp. 620-622, 1992.

[4] H. Watabe, V. J. Cunningham, A. A. Lammerstsma, P. M. Bloomfield, M. Mejia, R. T. Fujiwara, A. K. P. Jones, and T. Nakamura, "Noninvasive quantification of $\mathrm{rCBF}$ using positron emission tomography," Quantification of Brain Function Using PET, R. Myers, V. Cunningham, D. Bailey, and T. Jones, Eds. San Diego, CA: Academic, 1996, pp. 191-195.

[5] R. E. Carson, Y. Yan, and R. Shrager, "Absolute cerebral blood flow with ${ }^{15} \mathrm{O}$-water and PET: Determination without a measured input function," Quantification of Brain Function Using PET, R. Myers, V. Cunningham, D. Bailey, and T. Jones, Eds. San Diego, CA: Academic, 1996, pp. 185-190.

[6] E. Henze, S. C. Huang, O. Ratib, E. Hoffman, M. E. Phelps, and H. R. Schelbert, "Measurement of regional tissue and blood radiotracer concentrations from serial tomographic images of the heart," J. Nucl. Med., vol. 24, pp. 987-996, 1983.

[7] K. P. Lin, S. C. Huang, Y. Choi, R. Brunken, H. Schelbert, and M. E. Phelps, "A method for correction myocardium to blood pool spillover in dynamic cardiac PET FDG studies," J. Nucl. Med., vol. 33, p. 882, 1992.

[8] K. Chen, S. C. Huang, and D. C. Yu, "The effects of measurement errors in plasma radioactivity curve on parameter estimation in positron 
emission tomography," Phys. Med. Biol., vol. 36, pp. 1183-1200, 1991.

[9] D. Feng, S. C. Huang, and X. Wang, "Models for computer simulation studies of input functions for tracer kinetic modeling with positron emission tomography," Int. J. Biomed. Comput., vol. 32, pp. 95-110, 1993.

[10] G. D. Hutchins, J. E. Holden, R. A. Koeppe, J. R. Halama, S. J. Gatley, and R. J. Nickles, "Alternative approach to single-scan estimation of cerebral glucose metabolic rate using glucose analogs, with particular application to ischemia," J. Cereb. Blood Flow Metab., vol. 4, pp. 35-40, 1984.

[11] S. Takikawa, V. Dhawam, P. Spetsieris, W. Robeson, T. Chaly, R. Dahl, D. Margouleff, and D. Eidelberg, "Noninvasive quantitative fluorodeoxyglucose PET studies with an estimated input function derived from a population-based arterial blood curve," Radiology, vol. 188, pp. 131-136, 1993.

[12] R. L. Phillips, C. Y. Chen, D. F. Wong, and E. D. London, "An improved method to calculate cerebral metabolic rates of glucose using PET," $J$. Nucl. Med., vol. 36, pp. 1668-1679, 1995.

[13] D. Feng, X. Wang, and H. Yan, "A computer simulation study on the input function sampling schedules in tracer kinetic modeling with positron emission tomography (PET)," Comput. Methods Program. Biomed., vol. 45, pp. 175-186, 1994.

[14] D. Feng and X. Wang, "A computer simulation study on the effects of input function measurement noise in tracer kinetic modeling with positron emission tomography (PET)," Comput. Biol. Med., vol. 23, pp. 57-68, 1993

[15] D. Feng, X. Li, and S. C. Huang, "A new double modeling approach for dynamic cardiac PET studies using noise and spillover contaminated LV measurements," IEEE Trans. Biomed. Eng., vol. 43, pp. 319-327, Mar. 1996.

[16] D. Feng and X. Wang, "A method for biomedical system modeling and physiological parameter estimation using indirectly measured input functions," Int. J. Syst. Sci., vol. 26, pp. 723-739, 1995.

[17] L. Sokoloff, M. Reivich, C. Kennedy, M. H. DesRosiers, C. S. Patlak, K. D. Pettigrew, D. Kakurada, and M. Shinohara, "The $\left[{ }^{14} \mathrm{C}\right]$ deoxyglucose method for the measurement of local cerebral glucose utilization: Theory, procedure and normal values in the conscious and anesthetized albino rat," J. Neurochem., vol. 28, pp. 897-916, 1977.

[18] R. A. Hawkins, M. E. Phelps, and S. C. Huang, "Effects of temporal sampling, glucose metabolic rates, and disruptions of the blood-brain barrier on the FDG model with and without a vascular compartment: Studies in human brain tumors with PET," J. Cereb. Blood Flow Metab. vol. 6, pp. 170-183, 1986.

[19] K. Levenberg, "A method for the solution of certain nonlinear problems in least squares," Q. Appl. Math., vol. 2, pp. 164-168, 1944.

[20] D. W. Marquardt, "An algorithm for least squares estimation of nonlinear parameters," J. Soc. Ind. Appl. Math., vol. 2, pp. 431-441, 1963.

Dagan Feng (''88-M'88-SM'94), for a photograph and biography, see this issue, p. 228 .

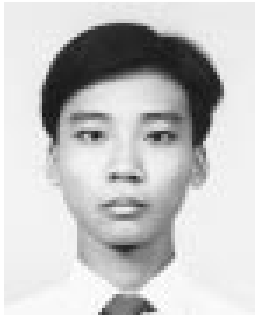

Koon-Pong Wong received the B.Eng. (honors) degree in electronic engineering from The Hong Kong Polytechnic University, Hung Hom, in 1996. $\mathrm{He}$ is currently pursuing the Ph.D. degree in the Department of Electronic Engineering, The Hong Kong Polytechnic University.

His research interests include computer modeling, signal processing, and analysis with biomedical applications.

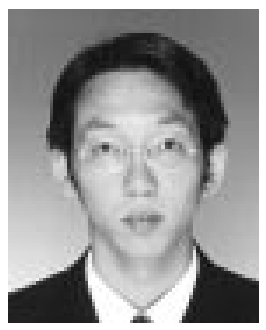

Chi-Ming Wu received the B.Eng. degree in electronic engineering from The Hong Kong Polytechnic University, Hung Hom, in 1996.

$\mathrm{He}$ is now working as an Engineer in industry.

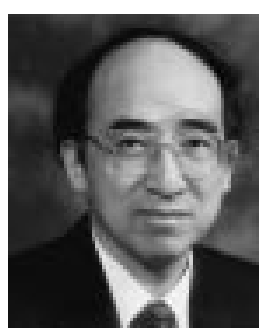

Wan-Chi Siu (M'77-SM'90) received the Associateship degree from The Hong Kong Polytechnic University, Hung Hom, the M.Phil. degree from the Chinese University of Hong Kong, and the Ph.D. degree from the Imperial College of Science, Technology and Medicine, London, U.K., in 1975, 1977, and 1984, respectively.

Between 1975 and 1980, he was with the Chinese University of Hong Kong and then joined The Hong Kong Polytechnic University in 1980 as a Lecturer became a Senior Lecturer in 1985, a Principal Lecturer in 1987, and a Reader in 1989. He subsequently became Chair Professor and Associate Dean of Engineering Faculty in 1992 and has been Chair Professor and Head of the Department of Electronic Engineering at the same university since 1994

Dr. Siu was an Associate Editor of the IEEE Transactions on Circuits AND SYSTEMS-PART II between 1995 and 1997 and has been a member of the Editorial Board of the Journal of VLSI Signal Processing Systems for Signal, Image, and Video Technology since 1996. He is also the Co-Guest Editor of the Special Issues in ISCAS' 97 of the IEEE TRANSACTIONS ON CIRCUITS AND SYSTEM —PART II, to be published in April 1998. He was the General Chairman of the International Symposium on Neural Networks, Image, and Speech Processing (ISSIPNN'94) and Co-Chair of the Technical Program Committee of the IEEE International Symposium on Circuits and Systems (ISCAS'97), which were held in Hong Kong in April 1994 and June 1997, respectively. In 1994, he chaired the first Engineering and Information Technology Panel to assess the research quality of 19 cost centers (departments) from all universities in Hong Kong. 